\section{Pain clinic referral to psychological services best addressed with collaboration}

\author{
Kathy Sexton-Radek, ${ }^{1}$ A. Tony Chami ${ }^{2}$ \\ 1Suburban Pulmonary and Sleep \\ Associates, Westmont, IL; ${ }^{2}$ Chicagoland \\ Advanced Pain Specialists, Chicago, IL \\ USA
}

\section{Abstract}

A case study of a middle aged female with severe musculoskeletal medical conditions causing severe pain is presented. The referral for psychological services was conducted in concordance with the medical treatments. In this case, the complex nature of the severe musculoskeletal medical conditions necessitated intensive care and the collaborative communications provided this to the patient. A tabulation of representative treatments is provided with an explanation of the nature of the collaboration. Outcome data in terms of patient self-reported pain ratings and sleep logging provided evidence of a mild improvement and stability where more negative findings, given the complexity of the medical conditions was expected.

\section{Introduction}

Collaborative inter-professional teamwork in health care including pain clinics has been increasing.1,2 The course of services is within the standard training of most Psychologists with concentrated training in Behavioral Medicine/ Medical Psychology.3,4 The range of Psychological services specific to the Pain Clinics entails assessment for treatment/medication abuse/functional status, treatments such as biofeedback, relaxation training, and psychotherapy. ${ }^{5}$ A case study is used to present the highlights of the collaboration between the Pain Clinic Physician and Psychologist. This case was a referral to psychological services from the pain clinic for assessment and treatment of the chronic pain with multiple disorders and disease diagnosis that the patient had been experiencing. Both extensive services and frequent professional communications provide the necessary level of care for complicated pain management cases..$^{4,6}$

\section{Case Report}

A fifty-four year old female presented to the
Pain Clinic with a general history of arthropathy pain for approximately ten years. The medical history consisted of the following: Lupus Erythematosus, Scleroderma, Raynaud's disease, Sarcodosis, Fibromyalgia, Rheumatoid Arthritis, and Osteoporosis that ranged from a two to ten year history. There was a surgical history of hip replacement, left pelvic bone reconstruction, thoracic disk degeneration release procedure. The patient presented with symptoms of low back and lower extremity pain, with muscle spasm, and myofascial pain. The spasms in the patient's legs had become involuntary secondary to little muscle control (i.e., involuntary spasms). The review of systems and neurological exams were negative per patient self-report. Patient was wheel chair bound for the last number of years as the back, hip and thigh pain was extreme and muscle tone was weak. Additionally, the spasms in her back were reported to extend to her hips, thighs and knees and prevented her walking and being in one position for longer than a thirty minute period of time. At intake, her Oswestry Disability Index score was $80 \%$. Following medication management that included hydrocodone and fentanyl patches and four sessions of cartilage replacement therapy, an Oswestry Disability Index of 58\% was reported.7,8 An 0swestry Disability Index was $32 \%$ following the implantation of temporary spinal cord stimulation trial lead bilaterally. The patient was taking minor tranquilizers at the maximum dosage/dosing previous to intake. She was counseled about the medication management protocol and endorsed the medication agreement and opioid consent form. The referral for Psychological treatment was made to setup ongoing supportive counseling, goal setting exercises, cognitive behavioral therapy and biofeedback approaches to address her pain condition. $3,9,10$ At intake, the Psychosocial Pain Inventory was used to identify the onset, duration, frequency, severity and location of the pain. Pain, prior to the intake was managed with a prescribed minor tranquilizer and active coping using her religious beliefs and practices.4,11 The patient was trained and worked as a professional nurse for some twenty years and possessed an understanding of her conditions. The patient's home had been modified for wheelchair passage, rails in the bathroom and in the common area on the first level that had been transferred into a bedroom. The patient was attended by family members on two week interval rotations. The patient's husband attended to the patient in the evening and weekends.3,4 The Strategies to Improve Motivation to Change, Pain Beliefs and Perceptions Inventory, and Oswestry Disability Index Scale were administered at intake. The findings from these scales indicated difficulty sleeping and a need to manage her time during the day. The patient was coop-
Correspondence: Kathy Sexton-Radek, Suburban Pulmonary and Sleep Associates, 700 E. Ogden Avenue, Westmont, Illinois 60559, USA.

Tel. +1.630.789.9785

E-mail: ksrsleep@aol.com

Key words: $\mathrm{AB}$ design, sleep quality, pain management, psychosocial treatment of pain.

Contributions: the authors contributed equally.

Conflict of interests: the authors report no conflict of interests.

Received for publication: 4 April 2013.

Revision received: 13 June 2013.

Accepted for publication: 15 June 2013.

This work is licensed under a Creative Commons Attribution NonCommercial 3.0 License (CC BYNC 3.0).

(C)Copyright K. Sexton-Radek and A.T. Chami, 2013 Licensee PAGEPress, Italy

Health Psychology Research 2013; 1:e33 doi:10.4082/hpr.2013.e33

erative with the Psychological intervention addressing these two areas.

\section{Discussion}

Using telephone and email communications, the Pain Clinic physician and Psychologist discussed the patient's needs and motivation to engage in medical and psychological treatments. Immediate communications through emails engaged a clear understanding between both professionals of their understanding of the case. Additionally, the concise, clear communications promoted a shared intervention that more broadly captured the progress of treatment (i.e., patient's understanding of her medical treatment was reviewed and addressed as well as her psychological reactions in the Psychology sessions). Each psychological treatment involved the focus on a treatment goal and included processing the adjustment to functioning and response to the medical treatment to alleviate pain. The precise management of patient progress and response lead to enhanced assessments of the patient's responses to treatment. In this manner, an awareness, at times, that additional explanations of treatment to address any misunderstandings or to manage resistance/poor compliance were managed efficiently.

The psychological assessment identified the patient's coping skills, perceptions of pain, and treatment goals. Findings indicated that the patient's affect and mood were within appro- 
Table 1. Collaboration with Medical and Psychological Treatments.

\begin{tabular}{|c|c|c|}
\hline Session & Medical procedure & Psychological treatment* \\
\hline 1 & $\begin{array}{l}\text { Medication management; cartilage replacement therapy treatment 1/4; } \\
\text { interference with activity }(60 \%) \text { and sleep ( } 85 \%) \\
\text { identified on visual analogue scale }\end{array}$ & Intake interview \\
\hline 2 & Discussed options of pain management & Activity management and pacing \\
\hline 3 & Insertion of temporary spinal stimulator & Activity management and pacing \\
\hline 4 & Temporary spinal stimulator & Mindfulness training; supportive psychotherapy \\
\hline 5 & Thoracolumbar brace; medication management & Mindfulness training; supportive psychotherapy \\
\hline 6 & Medication management; spinal adjustment & Biofeedback; supportive psychotherapy \\
\hline 7 & - & Biofeedback; supportive psychotherapy \\
\hline 8 & Pain management medication; steroid injection for knee joint & Biofeedback; supportive psychotherapy \\
\hline 9 & Pain management medication; steroid injection for knee joint & Supportive psychotherapy \\
\hline 10 & Ultrasound; dorsal column spinal stimulator; first medial branch procedure & Supportive psychotherapy \\
\hline 11 & $\begin{array}{l}\text { Dorsal column spinal stimulator; transverse process L3, L4, L5; } \\
\text { posterior lateral superior articulating process S1 injection }\end{array}$ & Supportive psychotherapy \\
\hline 12 & Unsuccessful left knee steroid injection; radio frequency denervation lumbar & Supportive psychotherapy \\
\hline 13 & Radio rhizotomy for left thigh pain & Supportive psychotherapy \\
\hline 14 & - & Teleconference; supportive psychotherapy \\
\hline
\end{tabular}

priate levels; she was appropriately sad and tearful when describing the pain and its extent. The treatment recommendations included the following themes: maintaining communications with patient's physicians about her condition(s), instructions in mindfulness and relaxation training techniques to manage pain, biofeedback treatments with home practices, activity, management and supportive psychotherapy. The patient was receptive to the recommendations and weekly sessions for a one year period ensued. Homework assignments such as logging activity were reviewed in sessions to emphasize the pacing behavior change goals that have been set up. The weekly visits ended as the patient's energy level and ability to attend the sessions with multiple medical appointments during her very small amount of alert/sufficient energy time (i.e., midmorning for about 90-110 minutes).

Sleep logs, pain diaries, and self-reported levels of activity satisfaction were used as outcome measures. Here again the communication between professionals provided immediate, visible data about the patient that was useful in treatment care and clinical understanding. The patient comments were favorable and seemed to have embedded a comfort with the continuity of care derived from the collaboration. The ordering of Psychological treatments was setup to address the alternate times when the patient was not directly in a pain treatment. This proved to provide comprehensive coverage to manage the pain for the patient.

The medical and psychological treatment pairings are listed in Table 1. Each psychological treatment involved the focus on a treatment goal and included processing the adjustment to functioning and response to the medical treatment to alleviate pain. Figure 1 depicts the
A

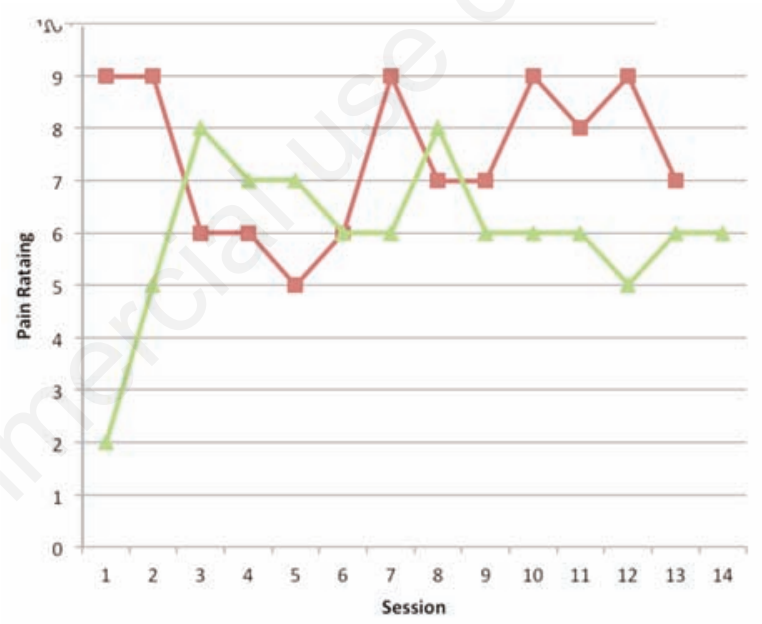

B

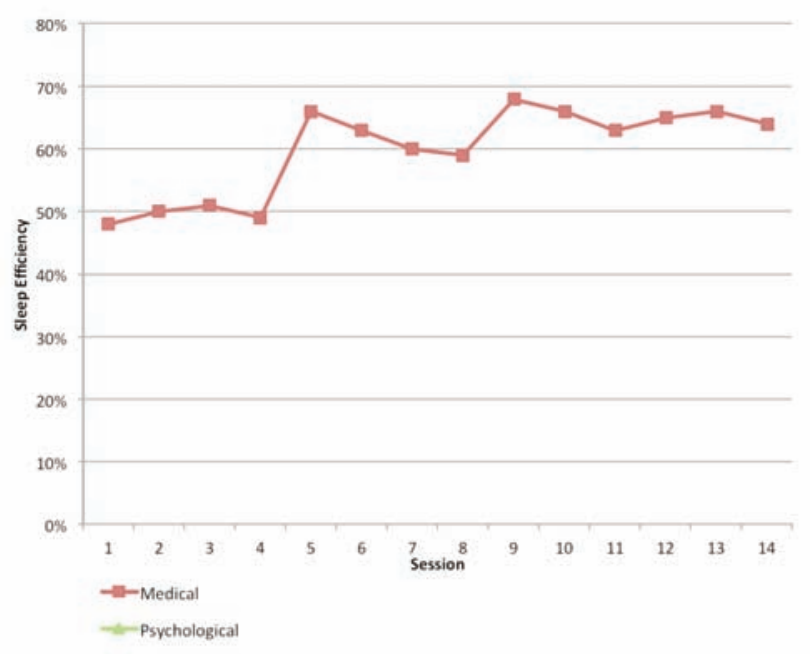

Figure 1. A) Pain ratings at time of treatment; B) sleep efficiency by session. Sleep efficiency is calculated from sleep logs where the amount of time asleep and in bed are recorded. The number of minutes asleep is divided by the number of minutes in bed and multiplied by one hundred to obtain the sleep efficiency value. A value of eighty-five percent is optimal; the higher the sleep efficiency value the better the sleep quality. This is a standard outcome variable in sleep medicine. 
change over time of the patient's pain ratings reported in each session. The visual analysis of Figure 1A illustrates some stability, on average, in the pain ratings given at the medical treatment times. However, within the context of the multiple medical diseases that the patient experienced individually, with each inducing a pain response, the stability and mild reduction in pain ratings is a success. ${ }^{12-14}$ In Figure 1B, the outcome variable, sleep efficiency is depicted. Over the course of treatment, the patient's sleep quality improved as indicated by the elevations in the sleep efficiency data. The achievement of the patient's response is believed to have been enhanced due to the comprehensive care of medical and psychological interventions.

\section{Conclusions}

The severity and complexity of the patient's condition were addressed with collaborative medical and psychological treatments. Although the patient's condition resulted in debilitation in musculoskeletal functioning, she was able to moderate her discomfort given with the medical treatments and psychological support. Her response and stable pain ratings with the medical treatment denoted this satisfactory response. The psychological gains are influenced by her moderately stable pain ratings and improved sleep quality. We concluded that the complexity of the patient's conditions which was to result in negative outcome was instead, stabilized (substantiated with positive patient feedback). The immediate, continuous clear communications about assessment, treatment, patient understanding and responses between the Psychologist and Physician at a Pain Clinic defined an effective collaboration.

\section{References}

1. Ahles TA, Wasson JH, Seville JA, et al. A controlled trial of methods of managing pain in primary care patients with or without co-occurring psychosocial problems. Ann Fam Med 2006;4:341-50.

2. Law B. Different skills, same team. Monitor Psychol 2013:40-3.

3. Blumenthal JA, McKee DC. Applications in behavioral medicine and health psychology: a clinician's source book. New York: Academic Press; 1987.

4. Sternback RA. Pain patient's traits and treatment. New York: Academic Press; 1987.

5. Von Korff M, Balderson BH, Saunders K, et al. A trial of an activity intervention for chronic back pain. Pain 2005;13:323-30.

6. Gerber L, el-Gabalawy H, Arayssi T, et al. Polyarticular arthritis, independent of rheumatoid factor, is associated with poor functional outcome in recent onset inflam- matory synovitis. J Back Musculoskelet Rehabil 2000;14:108-9.

7. Osteoporosis Quality of Life Study Group. Measures of quality of life in women with osteoporosis. Osteoporosis Int 1997;7:47887.

8. Gould E, Ma T. Back pain. Arch Phys Med Rehabil 2006;87:e38.

9. Kincade S. Evaluation and treatment of acute low back pain. Am Fam Phys 1991; 75:1181-92.

10. Gaston-Johansson F. Measurement of pain: the psychometric properties of the Pain-0-Meter, a simple, inexpensive pain assessment tool that could change health care practices. J Pain Symptom Manage 1996;12:172-81.

11. Brey RL, Petri MA. Neuropsychiatric systemic lupus erythematous: miles to go before we sleep. Neurology 2003;61:9-10.

12. Van Wijk RM, Geurts JW, Wynne HJ. Longlasting analgesia effect of radio frequency treatment of the lumbosacral dorsal root ganglion. J Neurosurg 2001;94 Suppl 2:227-31.

13. Sluijter ME, Cosman ER, Rittman WB, VanKleef M. The effects of pulsed radio frequency field applied to dorsal root ganglion - a preliminary report. Pain Clinic 1998;11:109-17.

14. Martin DD, Willis ML, Mullinax A, et al. Pulsed radio frequency applications in the treatment of chronic pain. Pain Practice 2007;7:31-5. 